\title{
Analysis of gene expression profiles associated with glioma progression
}

\author{
GUOZHANG HU ${ }^{1 *}$, BO WEI $^{2 *}$, LINA WANG $^{3}$, LE WANG $^{4}$, DALIANG KONG $^{1}$, YING JIN $^{5}$ and ZHIGANG SUN ${ }^{2,6}$ \\ Departments of ${ }^{1}$ Emergency Medicine, ${ }^{2}$ Neurosurgery and ${ }^{3}$ Ophthalmology, China-Japan Union Hospital of Jilin University, \\ Changchun, Jilin 130033; ${ }^{4}$ Department of Opthalmology, The First Hospital of Jilin University, Changchun, Jilin 130021; \\ ${ }^{5}$ Department of Neurology, Jilin Oilfield General Hospital, Songyuan 131200; ${ }^{6}$ Department of Neurosurgery, \\ Affiliated Hospital of Inner Mongolia University for The Nationalities, Inner Mongolia 028007, P.R. China
}

Received July 4, 2014; Accepted March 12, 2015

DOI: $10.3892 / \mathrm{mmr} .2015 .3583$

\begin{abstract}
The present study aimed to investigate changes at the transcript level that are associated with spontaneous astrocytoma progression, and further, to discover novel targets for glioma diagnosis and therapy. GSE4290 microarray data downloaded from Gene Expression Omnibus were used to identify the differentially expressed genes (DGEs) by significant analysis of microarray (SAM). The Short Time Series Expression Miner (STEM) method was then applied to class these DEGs based on their degrees of differentiation in the process of tumor progression. Finally, EnrichNet was used to perform the Kyoto Encyclopedia of Genes and Genomes (KEGG) pathway enrichment analysis based on a protein-protein interaction (PPI) network. A total of 4,506 DEGs were detected, and the number of DEGs was the highest in grade IV cells (2,580 DEGs). These DEGs were classified into nine clusters by the STEM method. In total, 11 KEGG pathways with XD-scores larger than the threshold $(0.96)$ were obtained. The DEGs enriched in pathways 1 (extracellular matrix-receptor interaction), 3 (phagosome) and 6 (type I diabetes mellitus) mainly belonged to cluster 5. Pathway 2 (long-term potentiation), 4 (Vibrio cholerae infection) and 5 (epithelial cell signaling in Helicobacter pylori infection) was involved with DEGs that belonged to different clusters. Significant changes in gene expression occurred during glioma progression. Pathways 1, 3 and 6 may be important for the deterioration of glioma into glioblastoma, and pathways 2, 4 and 5 may have a role at each stage during glioma progression. The
\end{abstract}

Correspondence to: Dr Zhigang Sun, Department of Neurosurgery, China-Japan Union Hospital of Jilin University, 126 Xiantai Avenue, Changchun, Jilin 130033, P.R. China

E-mail: sunzg001@126.com

*Contributed equally

Key words: glioma progression, gene expression profile, differentially expressed genes, kyoto encyclopedia of genes and genomes enrichment pathway associated DEGs, including $S V 2, N M D A R$ and $m G l u R s$, may be suitable as biomarkers or therapeutic targets for gliomas.

\section{Introduction}

Glioma is the most common and most aggressive malignant primary brain tumor in humans, with a morbidity rate of $6 / 100,000$ and a five-year survival rate of 20-30\% (1). Gliomas are classified into low-grade types (I or II) with slow or relatively slow growth, and high-grade types (III or IV), with fast growth and spread into normal brain tissue based on the World Health Organization (WHO) grading system (2). Patients with glioblastoma, the highest-grade glioma, survive for no more than one year following diagnosis, as there are still no efficient treatment protocols at present (3). Therefore, early diagnosis of gliomas is crucial for improving the survival of patients.

At present, diagnosis of gliomas is mainly based on histological detection, which, however, cannot reflect the pathological changes at the cellular and molecular levels timely and efficiently. Although the importance of numerous genes, including TP53, PTEN, EGFR, MDM2, CDKN2A and $C D K N 2 B$ (4-6), during glioma progression has been proven, assays for individual genes/proteins or in combination with histological features are neither predictive of survival of glioma patients nor able to guide therapeutic decisions (7). By constrast, microarrays can provide tremendous information at the transcription level, and are thus likely to reveal dynamic changes in the expression of multiple genes simultaneously. To date, microarray analysis has been successfully used to identify unknown tumor glioma subtypes (8), and gene-based classification has been proven to better correlate with patient survival than histological classification (9).

In the present study, microarray analysis data were subjected to bioinformatics analysis in order to investigate changes at the transcript level that are associated with spontaneous glioma progression, with the objective to discover novel targets for glioma diagnosis and therapy. First, gene expression profile data of glioma samples were compared with non-cancerous samples from epilepsy patients to detect the differentially expressed genes (DEGs) by the significance analysis of microarray (SAM) method. Then, the short time-series expression miner (STEM) method was applied to class these DEGs based on their degree 
of differentiation in the process of tumor progression. Finally, EnrichNet was used to analyze the enrichment of classified DEGs on Kyoto Encyclopedia of Genes and Genomes (KEGG) pathways based on a protein-protein interaction (PPI) network.

\section{Materials and methods}

Affymetrix microarray data. The expression profile of GSE4290 (10) was acquired from the Gene Expression Omnibus (GEO, http://www.ncbi.nlm.nih.gov/geo/) database. The platform of those data is GPL570 [HG-U133_Plus_2] Affymetrix Human Genome U133 Plus 2.0 Array. In total, 23 brain tissue samples from epilepsy patients as non-tumor (control) samples and 157 glioma samples, including 26 astrocytoma samples (7 grade II and 19 grade III), 50 oligodendroglioma samples (38 grade II and 12 grade III) and 81 glioblastoma samples (grade IV) were used.

Data pre-processing. First, the expression microarray datasets of the glioma and control samples were extracted using the R affy package (http://www.bioconductor.org/packages/release/bioc/html/affy.html). Then, the Robust Multiarray Average (RMA) method, practically supported by the justRMA function of the $\mathrm{R}$ affy package, was used to normalize the data through $\log 2$ transformation (11).

DEG screening and clustering. Significance analysis of microarray (SAM) of two classes of unpaired measurements was performed for DEG identification using the MeV software (12). Only genes with $\mid \log$ fold change (FC) $\mid \geq 2$ and a false detection rate $(\mathrm{FDR})<0.05$ were regarded as $\mathrm{DEG}$ between the control and the tumor samples. Regardless of the cell type, DEGs at three grades (II, III and IV) were obtained. Using the Short Time Series Expression Miner (STEM) method specifically designed for the analysis of short time series gene expression data by Ernst and Bar-Joseph (13), these DEGs were classified according to their differential expression degrees during tumor progression.

Construction of PPI network and KEGG pathway enrichment analysis. The web-accessible online bioinformatics tool EnrichNet (http://www.enrichnet.org/) was used to perform a functional enrichment analysis of the classified DEGs (14) through calculating the overlaps between KEGG pathway and PPI network built with the self-defined gene sets. Unlike the methods (e.g. WebGestalt, http://bioinfo. vanderbilt.edu/webgestalt/ and DAVID, http://david.abcc. ncifcrf.gov/) that only consider overlaps between self-defined gene sets and a known KEGG pathway gene and address the importance of overlaps based on the q-value by statistical tests (eg. one-sided Fisher's exact test), EnrichNet is an analytical method based on protein-protein interaction (PPI) networks. First, a PPI network was built using the Search Tool for the Retrieval of Interacting Genes (STRING) database (15). Then, by comparing the PPI network with the KEGG database, the similarity degree between PPI and a certain pathway was determined using EnrichNet, which is expressed as the XD-score, with a higher XD-score meaning a greater similarity, indicating higher probability of enrichment in a certain KEGG pathway. To help set the XD-score threshold, EnrichNet also calculated the significance score (q-value) by the classical overlap-based Fisher test, followed by linear regression analysis with $\mathrm{XD}$-score, by which the $\mathrm{XD}$-score corresponding to the q-value of 0.05 was selected as the threshold. Eventually, the obtained Pearson correlation coefficient was 0.85 , and the $\mathrm{XD}$-score was set at 0.96 .

\section{Results}

Screening of DEGs. Through microarray analysis, a total of 4,506 DEGs were detected and the number of DEGs increased with the increasing glioma grade, with the largest number of DEGs $(2,580)$ in grade IV cells. The top ten up- and downregulated DEGs at each grade were listed by their FC value (Table I).

Grade-dependent gene clustering. Several genes were not differentially expressed at all the three grades, and the missing expression value was assigned as 0. According to STEM analysis, the DEGs were grouped into nine important gene clusters, which were ranked according to their significance (P-value) (Table II). The differences in the expression values of almost all the DEGs increased with the increasing grade: DEGs in clusters 1, 6, 7, 8 and 9 revealed differential expressions at grade II, while DEGs in clusters 3 and 4 revealed differential expression at grade III, and DEGs in clusters 2 and 5 exhibited differential expression at grade IV (Fig. 1).

Pathway enrichment analysis of the PPI network. A total of 2,199 genes in the nine clusters were submitted to EnrichNet to construct a PPI network, followed by KEGG pathway enrichment analysis. Finally, 11 KEGG pathways with XD-scores larger than the threshold (0.96) were re-ranked according to their corresponding Fisher q-values (Table III). If q-value $<0.05$ was set as the threshold, only the first six pathways were enriched.

For pathway 1 [extracellular matrix (ECM)-receptor interaction] (Fig. 3), 3 (phagosome) and 6 (type I diabetes mellitus), genes participating in these three pathways largely belonged to cluster 5 . For example, the collagen-encoding genes (COL1A2, COL3A1, COL4A1 and COL6A2) and integrin-encoding genes (ITGA and ITGB) in pathways 1 and 3, and $H L A$ genes (HLA-DPA1, HLA-DPB1, HLA-DQB1, $H L A-D R A, H L A-D R B 1$ and $H L A-G)$ in pathways 3 and 6 may have important roles in the development progress of malignant gliomas. For pathways 2 (long-term potentiation) (Fig. 4), 4 (Vibrio cholerae infection) and 5 (epithelial cell signaling in Helicobacter pylori infection), genes involved in these three pathways belonged to different clusters, indicating that these genes may be associated with the occurrence of glioma. In particular, genes belonging to clusters 1, 8 and 9, which were differentially expressed in grade II glioma cells, accounted for nearly $50 \%$ of DEGs in pathway 2 (Fig. 2).

\section{Discussion}

In the present study, the DEGs enriched in pathways 1, 3 and 6 mainly belonged to cluster 5 and were differentially expressed at grade IV, indicating that these pathways and their associated DEGs may have important roles in the deterioration of glioma 
Table I. Top ten down- and upregulated differentially expressed genes in grade II, grade III and grade IV glioma samples.

\begin{tabular}{|c|c|c|c|}
\hline DEGs & Grade II & Grade III & Grade IV \\
\hline \multirow[t]{10}{*}{ Downregulated } & SERPINF1 & BRSK1 & PRCD \\
\hline & EPHA5 & MAFK & KIAA1324L \\
\hline & TMEFF2 & C6orf114 & KCNIP2 \\
\hline & FLJ30594 & LOC730125 & PPP2R2B \\
\hline & KIFC2 & SEC61A2 & TGFA \\
\hline & FEZF2 & BTBD9 & ARHGEF4 \\
\hline & STOX2 & $\mathrm{MEF} 2 \mathrm{C}$ & C10orf84 \\
\hline & AP1S1 & ENTPD4 & DPY19L2P2 \\
\hline & ADARB 1 & CACNB3 & TMEM16E \\
\hline & YWHAB & BRUNOL6 & PLCL2 \\
\hline \multirow[t]{10}{*}{ Upregulated } & TMEM100 & SOX11 & TOP2A \\
\hline & LPL & hCG_1815491 & IGFBP2 \\
\hline & MTHFD2 & TOP2A & PTX3 \\
\hline & SOX11 & LPL & hCG_1815491 \\
\hline & BMP2 & EGFR & POSTN \\
\hline & DLL3 & PBK & EGFR \\
\hline & C20orf42 & TMEM100 & MEOX2 \\
\hline & TIMP4 & RRM2 & NNMT \\
\hline & SOX4 & TMSL8 & RRM2 \\
\hline & EGFR & TIMP4 & IBSP \\
\hline
\end{tabular}

Table II. Differentially expressed genes clustered by the short time-series expression miner method.

\begin{tabular}{lcc}
\hline Cluster & Number of genes & P-value \\
\hline 1 & 269 & $1.60 \times 10^{-187}$ \\
2 & 374 & $2.30 \times 10^{-54}$ \\
3 & 296 & $5.20 \times 10^{-53}$ \\
4 & 264 & $8.00 \times 10^{-39}$ \\
5 & 478 & $3.70 \times 10^{-36}$ \\
6 & 36 & $2.80 \times 10^{-21}$ \\
7 & 32 & $1.20 \times 10^{-17}$ \\
8 & 127 & $1.40 \times 10^{-7}$ \\
9 & 323 & $6.30 \times 10^{-4}$ \\
\hline
\end{tabular}

into glioblastoma. Among these DEGs, integrin-encoding genes (ITGA and ITGB) were observed to be upregulated in pathways 1 and 3. Taking the ECM-receptor interaction pathway as an example, it refers to the interaction between ECM components and glioma cells, during which cellular matrix receptors function as mediators, and this interaction was previously reported to be responsible for the clinically important features of malignant gliomas, including cerebral invasion and leptomeningeal spread (16). Integrins, a major group of ECM receptors, which are involved in the adhesion and basement membrane invasion of glioma cells (16), were upregulated in this pathway. Collagen-encoding genes, which were markedly upregulated, took a large proportion of DEGs in the first pathway and mostly belonged to cluster 5, suggesting their important role in the deterioration of glioma into glioblastoma. Collagens are primary components in the ECM of most cell types and have been reported to be elevated in gliomas compared to those in normal adult brain cells as well as to have an important role in driving tumor progression (17). In this pathway, a small number of genes, including $S V 2$ ( $S V 2 B$ and $S V 2 C$ ), were differentially expressed very early at grade II. Synaptic vesicle glycoproteins (SV2; with the three different isoforms SV2A, SV2B and SV2C) are a group of integral transmembrane proteins (18) with an important role in the development of the central nervous system $(19,20)$. According to a study on patients orally administrated with levetiracetam following surgery, an obvious increase in $S V 2$ expression was observed in those with a good response (21), suggesting that higher $S V 2$ levels may indicate a good prognosis. Thus, the significant decrease in $S V 2$ expression at grade II in this pathway was speculated to correlate with the occurrence of glioma. In addition, human leukocyte antigen (HLA) genes, including HLA-DPA1, HLA-DPB1, $H L A-D Q B 1, H L A-D R A, H L A-D R B 1$ and $H L A-G$, took a large proportion of DEGs in either pathway 3 or 6 , particularly in pathway 6 , in which these genes were upregulated at grade IV, conforming to the significant expression of $H L A-D Q B 1$ and $H L A-D R B 1$ in patients with high-grade glioma (HGG) observed by La Torre et al (22), and the positive correlation between $H L A-D R B 1$ and symptomatic cerebral glioma reported by Guerini et al (23). Furthermore, introduction of HLA-Gl or HLA-G5 into HLA-G-negative glioma cells (U87MG) rendered them highly resistant to direct alloreactive lysis, inhibited the alloproliferative response and prevented efficient priming of cytotoxic T cells (24); thus, this gene may contribute to the immune escape in human glioblastoma. 
Table III. Enriched Kyoto Encyclopedia of Genes and Genomes pathways for differentially expressed genes in the protein-protein interaction network.

\begin{tabular}{clccc}
\hline Rank & \multicolumn{1}{c}{ Pathway } & XD-score & Fisher q-value & $\begin{array}{c}\text { Overlapping gene } \\
\text { number }\end{array}$ \\
\hline 1 & \multicolumn{1}{c}{ hsa04512: Extracellular matrix-receptor interaction } & 1.7601 & $7.58 \times 10^{-6}$ & 29 \\
2 & hsa04720: Long-term potentiation & 1.6560 & $4.84 \times 10^{-4}$ & 22 \\
3 & hsa04145: Phagosome & 1.0851 & $4.95 \times 10^{-4}$ & 36 \\
4 & hsa05110: Vibrio cholerae infection & 1.9504 & $6.95 \times 10^{-4}$ & 18 \\
5 & hsa05120: Epithelial cell signaling in & 1.1594 & $4.78 \times 10^{-3}$ & 19 \\
& Helicobacter pylori infection & & & 13 \\
7 & hsa04940: Type I diabetes mellitus & 1.3101 & $1.69 \times 10^{-2}$ & 10 \\
8 & hsa03030: DNA replication & 1.3351 & $6.49 \times 10^{-2}$ & 7 \\
9 & hsa04964: Proximal tubule bicarbonate reclamation & 1.8351 & $6.77 \times 10^{-2}$ & 7 \\
10 & hsa04320: Dorso-ventral axis formation & 1.1829 & $1.17 \times 10^{-1}$ & 5 \\
\hline
\end{tabular}
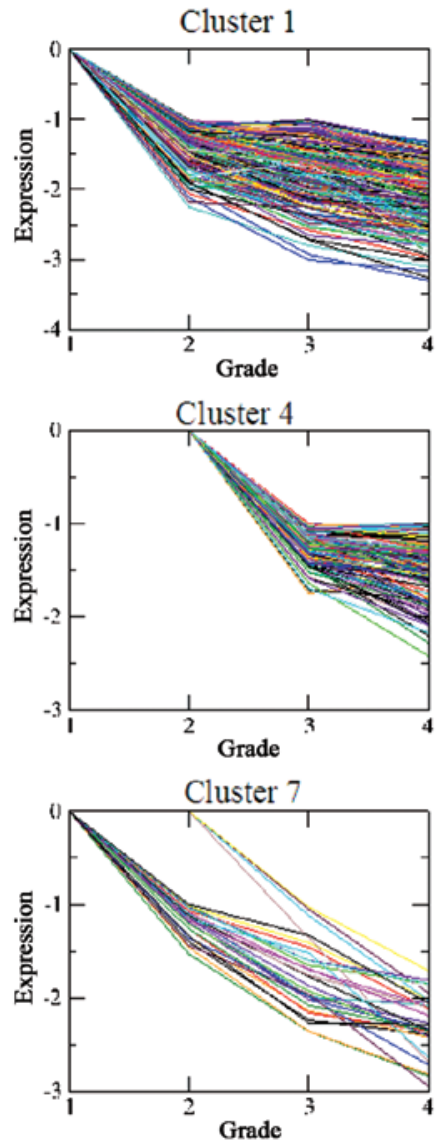

Cluster 2
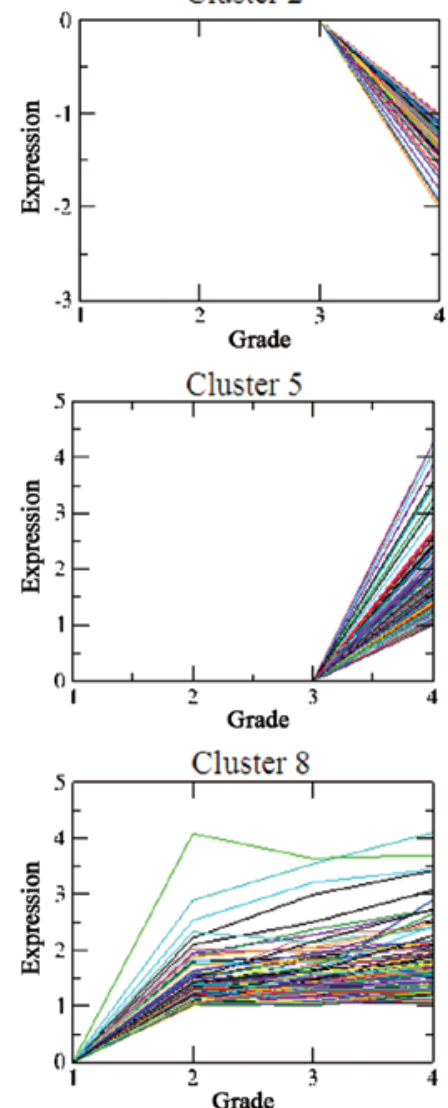

Cluster 3

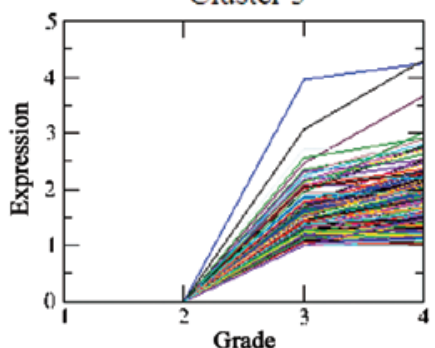

Cluster 6

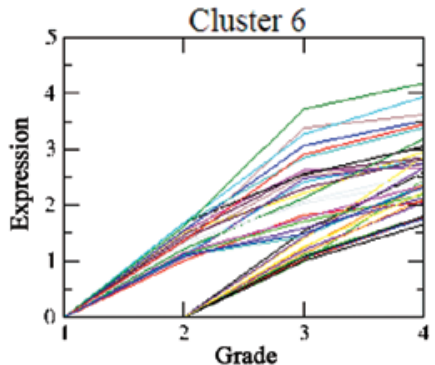

Cluster 9

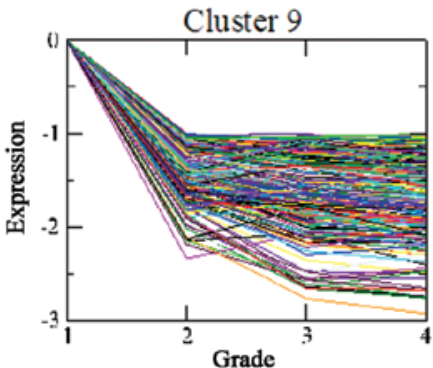

Figure 1. Gene clustering by short time series expression miner analysis. Each line in the figure represents an expression value of the corresponding gene. The abscissa represents the grade of glioma, and the ordinate represents the $\log _{2}$ value of the fold change. Negative values indicate downregulated expression, and positive values indicate upregulated expression.

Furthermore, DEGs exclusively detected at grade IV with large $\mathrm{FC}$ values (i.e., IGFBP2 and GABRA5) revealed huge variation in expression levels compared to previous grades, which was speculated to be closely correlated with the significant changes during the development of glioma into glioblastoma. IGFBP2 was reported to be overexpressed in glioblastoma and promote glioma tumor stem cell expansion and survival (25). Gamma-aminobutyric acid (GABA) A receptor, alpha 5 (GABRA5) (26) is a part of the extrasynaptic GABA A-channels and involved in tonic currents (27). The lowest GABRA5 mRNA levels were found in glioblastoma compared to gliomas of lower malignancy (28), which is 

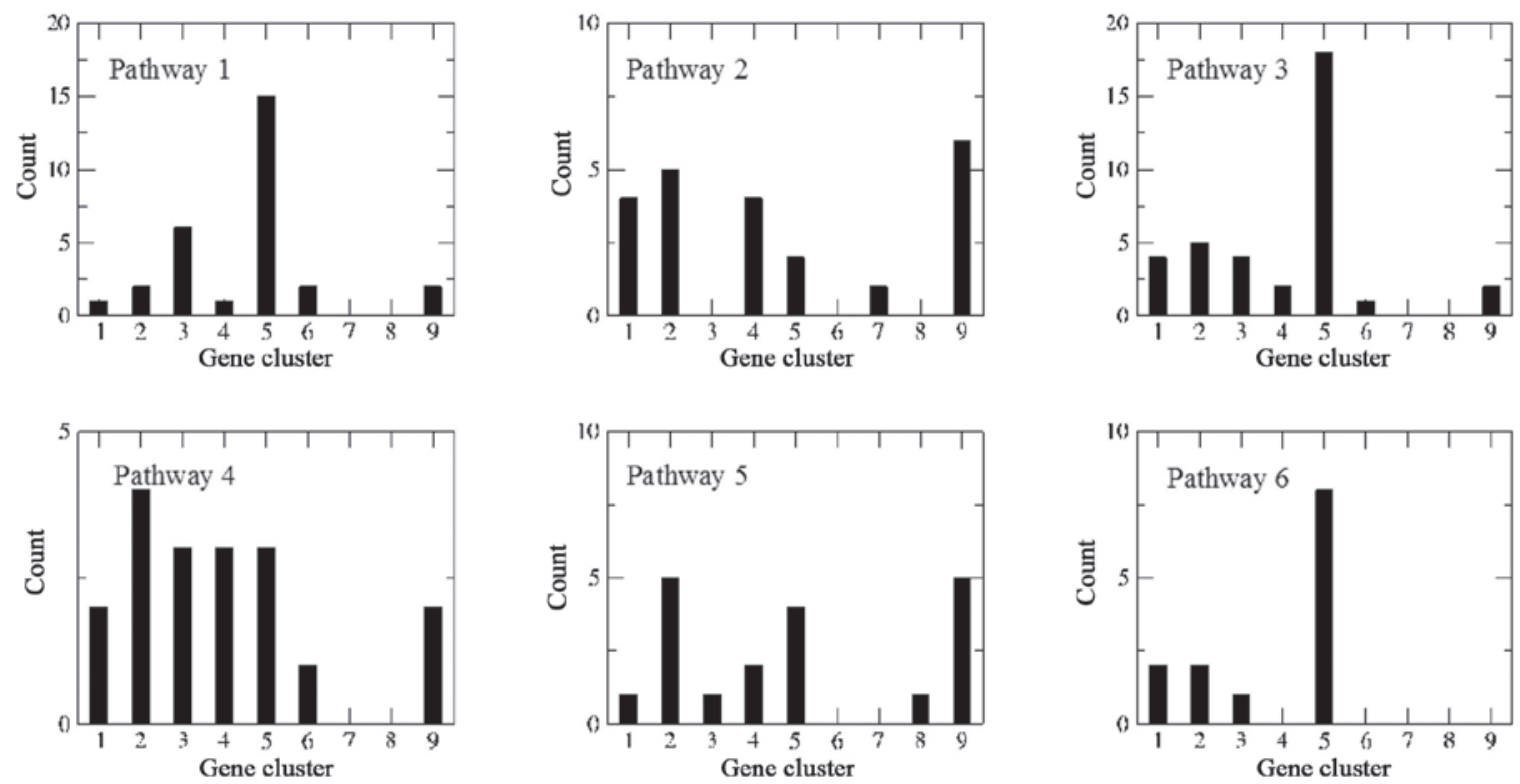

Figure 2. Statistics of overlapping genes between Kyoto Encyclopedia of Genes and Genomes pathways and differentially expressed genes in clusters.

\section{ECM-RECEPTOR INTERACTION}
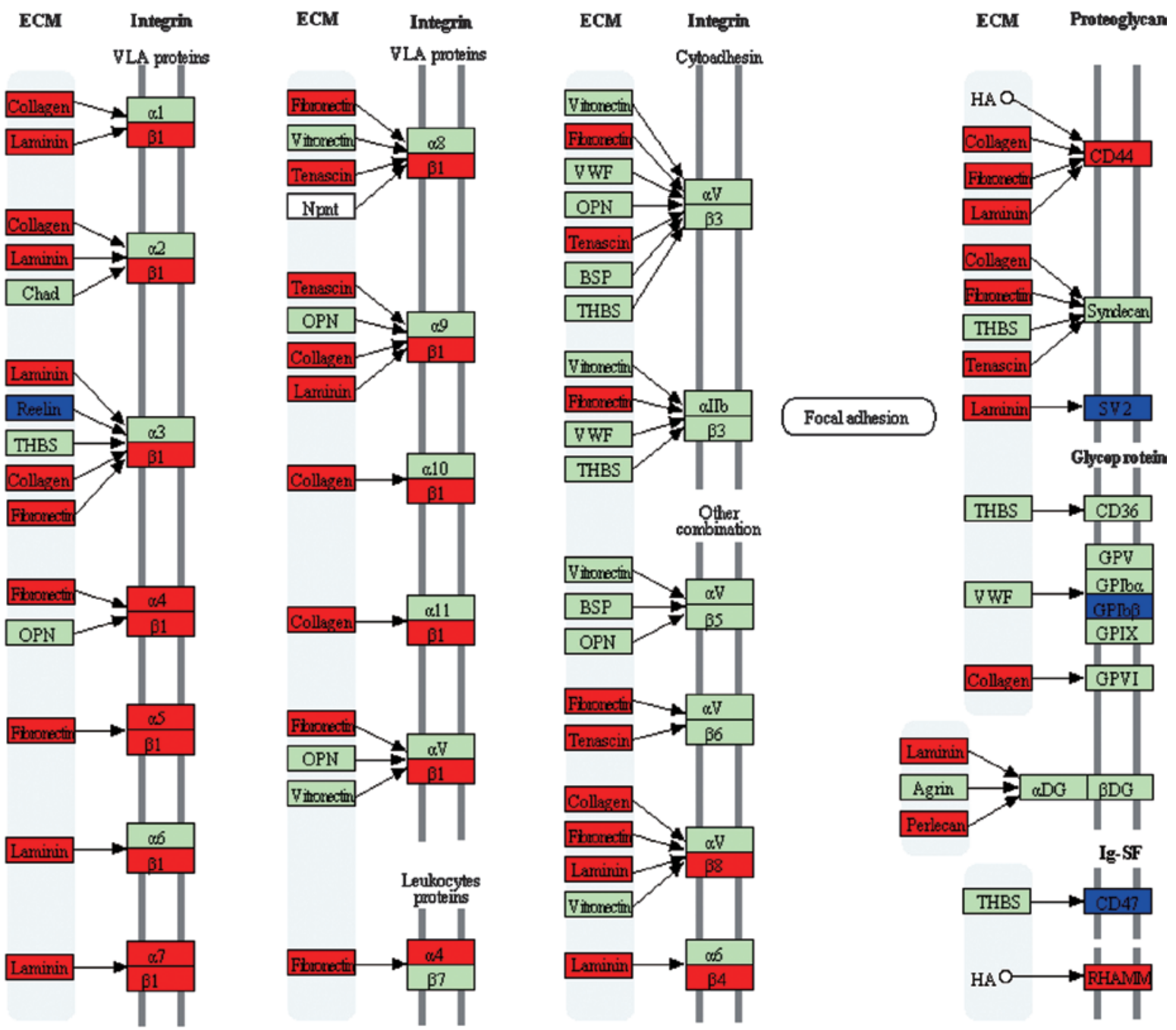
LONG-TERM POTENTIATION

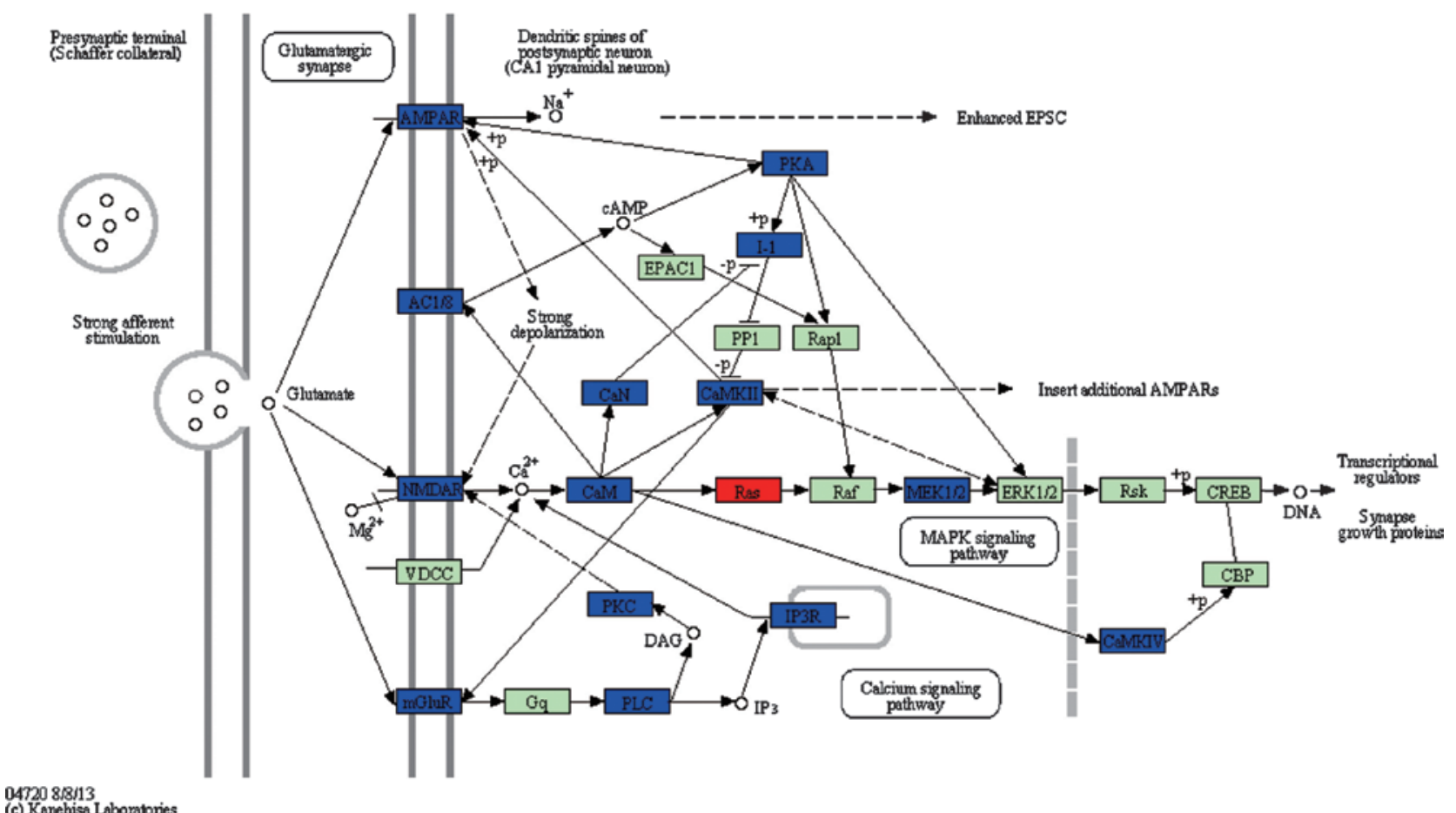

Figure 4. hsa04720: Long-term potentiation (red color indicates upregulated genes and blue color indicates downregulated genes)

consistent with the remarkable decrease in GABRA5 expression.

Since the DEGs participating in pathways 2,4 and 5 belong to different clusters, it can be inferred that these pathways and their associated DEGs may have a role at each stage during glioma progression. As the long-term potentiation pathway contained a large proportion of DEGs from clusters 1,8 and 9, which were differentially expressed in very low-grade glioma cells, it is presumed that this pathway is closely associated with the occurrence of glioma. This pathway has been widely considered as one of the major cellular mechanisms that underlies learning and memory (29). Memory reduction is the most important and distinctive feature in high-grade glioma, thus it can be inferred that memory impairment in high-grade glioma patients is associated with the pathological changes in this molecular process, which may be attributed to the alterations in gene expression in this pathway. Compared to changes observed by Dong et al (30) in the LTP enrichment pathway, there were no significant changes observed in the expression of PP1, Rapl, Raf and ERK1/2, with downregulation of I-1, $A M P A R$ and $A C 1 / 8$ expression, and upregulation of Ras in the present study. The difference in the expression of these genes may be attributed to the difference in sample size, and should be further investigated. Additionally, Dong et al (30) observed the downregulation of $m G l u R 5$ expression in this pathway at grade II, which is consistent with a previous study that reported the impaired learning and reduced CA1 LTP in mice lacking mGluR5 (31).

In conclusion, long-term potentiation and ECM-receptor interaction were discovered to have an important role in the occurrence and development of gliomas, which may provide a novel and comprehensive view for the treatment of gliomas. The associated DEGs, including SV2, NMDAR and $m G l u R s$, can be considered to be used as biomarkers or therapeutic targets for gliomas. However, the results of the present study require to be further confirmed by additional experiments.

\section{References}

1. Mamelak AN and Jacoby DB: Targeted delivery of antitumoral therapy to glioma and other malignancies with synthetic chlorotoxin (TM-601). Expert Opin Drug Deliv 4: 175-186, 2007.

2. Goebell E, Paustenbach S, Vaeterlein O, et al: Low-grade and anaplastic gliomas: differences in architecture evaluated with diffusion-tensor MR imaging. Radiology 239: 217-222, 2006.

3. Fine HA, Dear KB, Loeffler JS, Black PM and Canellos GP: Meta-analysis of radiation therapy with and without adjuvant chemotherapy for malignant gliomas in adults. Cancer 71: 2585-2597, 1993.

4. Stark AM, Hugo H, Witzel P, Mihajlovic Z and Mehdorn HM: Age-related expression of p53, Mdm2, EGFR and Msh2 in glioblastoma multiforme. Zentralbl Neurochir 64: 30-36, 2003.

5. Li J, Yen C, Liaw D, et al: PTEN, a putative protein tyrosine phosphatase gene mutated in human brain, breast and prostate cancer. Science 275: 1943-1947, 1997.

6. Simon M, Köster G, Menon AG and Schramm J: Functional evidence for a role of combined CDKN2A (p16-p14ARF)/CDKN2B (p15) gene inactivation in malignant gliomas. Acta Neuropathol 98: 444-452, 1999.

7. Freije WA, Castro-Vargas FE, Fang Z, et al: Gene expression profiling of gliomas strongly predicts survival. Cancer Res 64: 6503-6510, 2004.

8. Shai R, Shi T, Kremen TJ, et al: Gene expression profiling identifies molecular subtypes of gliomas. Oncogene 22: 4918-4923, 2003.

9. Nutt CL, Mani D, Betensky RA, et al: Gene expression-based classification of malignant gliomas correlates better with survival than histological classification. Cancer Res 63: 1602-1607, 2003.

10. Sun L, Hui AM, Su Q, et al: Neuronal and glioma-derived stem cell factor induces angiogenesis within the brain. Cancer Cell 9: 287-300, 2006 
11. Gruber HE, Ingram JA, Hoelscher GL, Zinchenko N, Hanley EN $\mathrm{Jr}$ and Sun Y: Asporin, a susceptibility gene in osteoarthritis, is expressed at higher levels in the more degenerate human intervertebral disc. Arthritis Res Ther 11: R47, 2009.

12. Larsson $\mathrm{O}$, Wahlestedt $\mathrm{C}$ and Timmons JA: Considerations when using the significance analysis of microarrays (SAM) algorithm. BMC Bioinformatics 6: 129, 2005.

13. Ernst J and Bar-Joseph Z: STEM: a tool for the analysis of short time series gene expression data. BMC Bioinformatics 7: 191, 2006.

14. Glaab E, Baudot A, Krasnogor N, Schneider R and Valencia A: EnrichNet: network-based gene set enrichment analysis. Bioinformatics 28: i451-i457, 2012.

15. Szklarczyk D, Franceschini A, Kuhn M, et al: The STRING database in 2011: functional interaction networks of proteins, globally integrated and scored. Nucleic Acids Res 39 (Database Issue): 561-568, 2011.

16. Paulus $\mathrm{W}$ and Tonn JC: Interactions of glioma cells and extracellular matrix. J Neurooncol 24: 87-91, 1995.

17. Payne LS and Huang PH: The pathobiology of collagens in glioma. Mol Cancer Res 11: 1129-1140, 2013.

18. Bajjalieh SM, Peterson K, Shinghal R and Scheller RH: SV2, a brain synaptic vesicle protein homologous to bacterial transporters. Science 257: 1271-1273, 1992.

19. Dong M, Yeh F, Tepp WH, et al: SV2 is the protein receptor for botulinum neurotoxin A. Science 312: 592-596, 2006.

20. Crèvecœur J, Foerch P, Doupagne M, et al: Expression of SV2 isoforms during rodent brain development. BMC Neurosci 14 $87,2013$.

21. de Groot M, Aronica E, Heimans JJ and Reijneveld JC: Synaptic vesicle protein $2 \mathrm{~A}$ predicts response to levetiracetam in patients with glioma. Neurology 77: 532-539, 2011.
22. La Torre D, Maugeri R, Angileri FF, et al: Human leukocyte antigen frequency in human high-grade gliomas: a case-control study in Sicily. Neurosurgery 64: 1082-1088, 2009.

23. Guerini FR, Agliardi C, Zanzottera M, et al: Human leukocyte antigen distribution analysis in North Italian brain glioma patients: an association with HLA-DRB1*14. J Neurooncol 77: 213-217, 2006.

24. Wiendl H, Mitsdoerffer M, Hofmeister V, et al: A functional role of HLA-G expression in human gliomas: an alternative strategy of immune escape. J Immunol 168: 4772-4780, 2002.

25. Hsieh D, Hsieh A, Stea B and Ellsworth R: IGFBP2 promotes glioma tumor stem cell expansion and survival. Biochem Biophys Res Commun 397: 367-372, 2010.

26. Otani K, Ujike H, Tanaka Y, et al: The GABA type A receptor a5 subunit gene is associated with bipolar I disorder. Neurosci Lett 381: 108-113, 2005.

27. Belelli D, Harrison NL, Maguire J, Macdonald RL, Walker MC and Cope DW: Extrasynaptic GABAA receptors: form, pharmacology and function. J Neurosci 29: 12757-12763, 2009.

28. Smits A, Jin Z, Elsir T, et al: GABA-A channel subunit expression in human glioma correlates with tumor histology and clinical outcome. PLoS One 7: e37041, 2012.

29. Bliss TV and Collingridge GL: A synaptic model of memory: long-term potentiation in the hippocampus. Nature 361: 31-39, 1993.

30. Dong H, Siu H, Luo L, Fang X, Jin L and Xiong M: Investigation gene and microRNA expression in glioblastoma. BMC Genomics 11 (Suppl 3): 16, 2010.

31. Lu YM, Jia Z, Janus C, et al: Mice lacking metabotropic glutamate receptor 5 show impaired learning and reduced CA1 long-term potentiation (LTP) but normal CA3 LTP. J Neurosci 17: 5196-5205, 1997. 